\title{
Temporal development of the behavioral effects of herpes encephalitis in mice
}

\author{
DENNIS J. MCFARLAND \\ New York State Department of Health, Albany, New York
}

\begin{abstract}
The temporal development of behavioral effects due to central nervous system infection with the herpes simplex type 1 virus was examined in two mouse models. Following infection of adult female NYA/Nylar mice with the HF strain of herpes, or adult female BALB/c mice with the F strain, the majority of animals survived. An increase in motor activity observed 7 days following infection of NYA/Nylar mice coincided in time with a decline in brain virus titers. Likewise, an increase in errors occurring during serial-reversal performance in a water Y-maze was observed 8 days following infection of Balb/c mice and coincided with the declining phase of the viral growth curve. Taken together, these results suggest that processes involved in the elimination of the virus from the brain, such as the cellular immune response, may be important in the development of behavioral effects produced by nonfatal herpes encephalitis.
\end{abstract}

A variety of model systems have been used to examine the development of virus-induced pathology. Cell-culture models are currently popular in the field of virology (e.g., Heeg, Dienes, Muller, \& Falke, 1986), and in the case of neurotropic viruses, cell lines with neuronal properties have been used (e.g., Rubenstein \& Price, 1983). Although such simplified systems may at times aid in the analysis of the nature of the pathological process, in vivo systems offer an added advantage when factors such as the immune response of the host are considered. The impact of virus infection may be indexed by parameters such as biochemical alterations in the target organ system (e.g., Seegal \& McFarland, 1988). However, the consequences of virus infection on the functioning of the target organ system represents an index that is more meaningful to the survival of the organism. In the case of neurotropic viruses, the target organ system is the brain and the appropriate functional index is the behavior of the organism.

The herpes simplex type 1 virus (HSV) is one of the more common causes of sporadic viral encephalitis in the human population and is noted for severe neurobehavioral disturbances (Adams \& Victor, 1981). Of these, a postencephalitic amnesic syndrome is perhaps best known (Hierons, Janota, \& Corsellis, 1978). Behavioral effects of HSV infection have been examined in several animal model systems (Lycke \& Roos, 1974; McFarland \& Hotchin, 1983; Seegal \& Hotchin, 1978).

Lycke and Roos (1974) examined young mice inoculated with fatal strains of HSV. They observed a gradual increase in the level of motor activity starting on the second day after intracerebral inoculaton and continuing until death occurred starting on the fourth day postinoculation.

I would like to thank R. F. Seegal for his valuable comments on an earlier draft of this manuscript. Correspondence may be addressed to Dennis J. McFarland, Wadsworth Center for Laboratories and Research, New York State Department of Mental Health, Albany, NY 12201-0509.
There is a considerable degree of variation in mortality and the rate of virus growth in the brain following infection with different strains of HSV (Dix, McKendall, \& Baringer, 1983). Although it is very likely that very high brain virus titers were rapidly reached in the study by Lycke and Roos (1974), this information was not reported in that publication. Accordingly, the present series of experiments was conducted to determine the evolution of behavioral effects and the corresponding rate of virus growth in two nonfatal models of HSV encephalitis. Two distinct virus-strain mouse-strain combinations were used since other aspects of the behavioral effects of HSV have previously been shown to be strain-dependent (McFarland \& Hotchin, 1983).

\section{EXPERIMENT}

\section{Method}

Subjects. The subjects were female NYA/Nylar mice approximately 90 days of age at the initiation of the experiment and obtained from the New York State Health Department breeding facilities. The animals were housed in plastic suspended cages with woodchip bedding, given food and water ad lib and maintained under diurnal lighting conditions ( $12 \mathrm{~h}$ on, $12 \mathrm{~h}$ off). The experiments were conducted in the middle $6 \mathrm{~h}$ of the lights-on cycle. The animals were inoculated with the virus suspension or diluent by first lightly anesthetizing them with ether and then injecting a $0.03-\mathrm{ml}$ volume of fluid intracerebrally. This intracerebral inoculation procedure, as standardly employed in virology, involves the hand-guided rapid introduction of a relatively large volume of fluid, which results in most of the fluid going into the ventricles and, to some extent, the subarachnoid space (McFarland \& Hotchin, 1987).

Virus. The HF strain of herpes simplex type 1 was obtained from the American Type Culture Collection and was grown in human embryonic lung cells. Viral titers were determined by a standard vero-cell plaque assay. Briefly, the cells were grown to near confluence in $60 \times 15 \mathrm{~mm}$ Petri dishes. Next, as much fluid as possible was removed and a 0.1 -ml sample from serial 10 -fold dilutions was added and allowed to incubate for $30 \mathrm{~min}$. Next, a mixture of $1 \%$ agarose and Eagle's medium at $40^{\circ} \mathrm{C}$ was added and the 
dishes were returned to the incubator. The agarose hardened at the $37^{\circ} \mathrm{C}$ temperature of the incubator and prevented the diffusion of the virus in media so that infection would spread only from cell to adjacent cell. After $48 \mathrm{~h}$, the cells were stained with Neutral Red and the number of virus plaques was counted. Since a vital stain was used, the number of foci of dead cells could be visualized. Virus titers were expressed as plaque-forming units (pfu). Since the number of assays required multiple nuns, only one sample from any given time-point was represented in each batch of assays.

Apparatus and Procedure. Activity levels were monitored individually for a 20 -min period at $2,4,8,16$, or 32 days following inoculation. A given animal was evaluated on only 1 day postinoculation. The animals were placed in $43 \times 24 \times 20 \mathrm{~cm}$ plastic cages painted flat black except for two small areas where two photocell units trisected the cage. Recording of the data was done with the aid of an ACT/interact laboratory microcomputer. The system was programmed so that interruptions of each beam were counted only once prior to the other beam being interrupted. This ensured that the system was measuring locomotion rather than small movements such as scratching or head movements in the path of a single photocell.

Brain homogenates were prepared by euthanizing a subset of the animals that had completed activity testing with ether, and rapidly removing the whole brain which was then frozen at $-70^{\circ} \mathrm{C}$. The brains were later thawed, and a $10 \%$ homogenate was made in Hank's balanced salt solution with $0.05 \%$ gelatin, penicillin, streptomycin, and Nystatin added to stabilize the virus, suppress bacteria, and suppress fungi, respectively. Homogenates were made with Ten Broeck-type glass homogenizers in an ice-water bath. The material was centrifuged for $5 \mathrm{~min}$ at $500 \mathrm{~g}$ and the supernate was used in the viral plaque assay.

The experiment was run in two replications. Animals from both replications were tested for activity levels in photocell cages. The first replication consisted of 30 vehicle-inoculated animals and 60 animals infected with 300 pfu of the HF strain of HSV. Following testing, animals in the first replication were monitored until death occurred or $\mathbf{5 0}$ days had passed since inoculation. The second replication consisted of 15 control and $\mathbf{4 0}$ infected subjects. Infected animals in the second replication were immediately used for virus assays. All control subjects were run in the activity-testing paradigm. Of the subjects infected with HSV, 35 from the first replication and 25 from the second replication were used in activity testing. Due to a death rate slightly higher than expected, subjects at 16 and 32 days postinfection were somewhat overrepresented in the second replication.

\section{Results}

Data on mortality are presented in Table 1 . Here it can be seen that no deaths occurred before 4 days postinfection and that the period from 5 to 8 days postinfection was associated with the highest mortality rate. If it was observed that an animal would not survive, that animal was euthanized by a lethal dose of pentobarbital.

Table 1

Mortality in NYA/Nylar Mice as a Function of Time

\begin{tabular}{cccc}
\hline Day & $\begin{array}{c}\text { Number of } \\
\text { Mice Dying }\end{array}$ & $\begin{array}{c}\text { Number of } \\
\text { Mice at Risk }\end{array}$ & $\begin{array}{c}\text { Mortality } \\
\text { (percent/day) }\end{array}$ \\
\hline $1-2$ & 0 & 60 & 0.00 \\
$3-4$ & 0 & 60 & 0.00 \\
$5-8$ & 5 & 60 & 2.08 \\
$9-16$ & 7 & 55 & 1.59 \\
$17-32$ & 2 & 48 & 0.26 \\
\hline
\end{tabular}

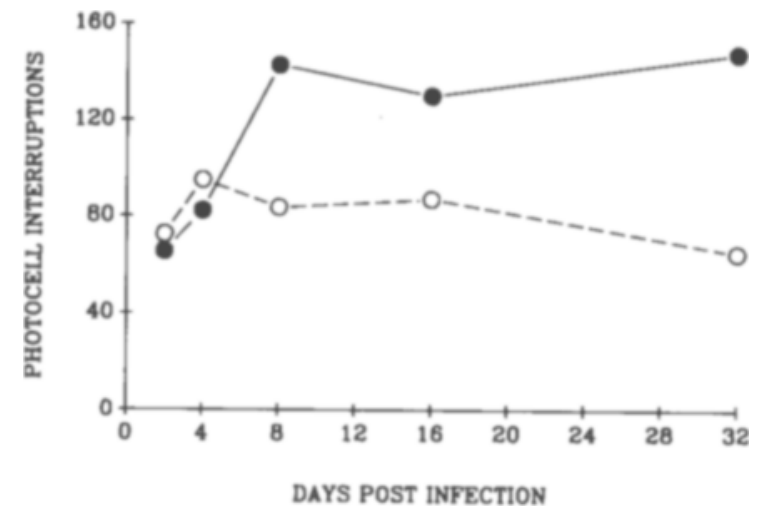

Figure 1. Photocell interruptions by NYA/Nylar mice infected with HSV (solid lines) and control mice (dashed lines) as a function of days following inoculation.

Data on activity levels are presented in Figure 1. The overall means for control animals in Replications 1 and 2 were 78.9 and 83.7 counts, respectively. Since the number of control subjects was proportionate across time periods, an analysis of variance (ANOVA) was conducted, which indicated that replications were not significant either as a main effect or as an interaction with days. A similar analysis for infected subjects was not possible since there was a confounding of days with replications in this group. Each point in Figure 1 represents the mean of 9 control or 12 infected animals. An ANOVA with both treatment and time period as between-subjects effects indicated that the effect of treatment $[F(1,95)=7.96 . p<.01]$ and the interaction between treatment and time period $[F(4,95)$ $=2.55, p<.05]$ were significant. Post hoc analysis (least-significant-difference test) indicated that the treatment effects were not significantly different prior to Day 8, after which they remained significantly different $(p<.01$ in all cases).

Data on the rate of viral growth are presented in Figure 2. Each point represents the mean of 5 or 6 animals. Here it can be seen that the peak viral titer occurred on Day 4 and thereafter declined. An ANOVA on the log values of the plaque count +1 indicated that the effects of time period $[F(3,19)=13.53, p<.0001]$ were significant.

These data indicate that with this particular model, both mortality rate and the behavioral effect examined are associated with the declining phase of the viral growth curve. This suggests that processes such as the immune response to infection may be producing these effects.

\section{EXPERIMENT 2}

\section{Method}

Subjects. The subjects were 8 control and 8 infected female Balb/c mice approximately 90 days of age at the start of the experiment. The animals were obtained from the New York State Health Department breeding facilities and maintained as in Experiment 1. Experimental subjects were infected with 100 pfu of the F strain of HSV, which was obtained from the American Type Culture Collection and prepared as was the virus strain in Experiment 1. 


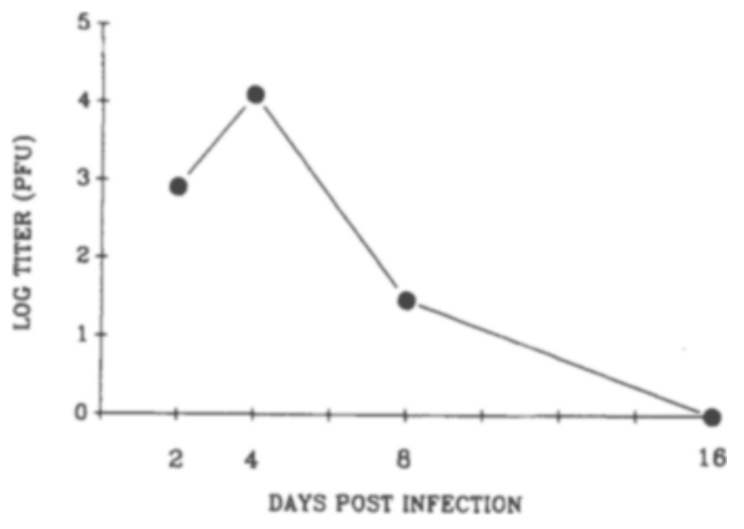

Figure 2. Brain virus titers in NYANylar mice infected with HSV as a function of days following inoculation.

Apparatus and Procedure. Prior to inoculation, the animals received 13 training sessions on a serial spatial-reversal task run in a water maze with one reversal per day. Each of these animals was then tested on the spatial-reversal task at 2, 4, 7, 10, 14, and 18 days postinoculation, with each animal being tested on each day postinfection. A separate set of 16 infected animals, which were not used for behavioral testing, were used for the determination of viral growth in the brain. Virus inoculation and brain titer determinations were done in a manner identical to that reported in Experiment 1 .

The maze was a $27.5 \times 20 \times 10 \mathrm{~cm}$ symmetrical Plexiglas $\mathrm{Y}$ shaped structure with L-shaped $10 \times 20 \times 10 \mathrm{~cm}$ goalboxes attached to the two choice arms. The correct arm contained a wire ladder leading to a holding cage. This procedure eliminated the need for handling, which greatly retards acquisition in this situation. The maze was filled to $12 \mathrm{~cm}$ with water. Any trial on which the subject entered the incorrect choice arm further than $15 \mathrm{~cm}$ was scored as an error. At that distance, the subject could not see whether the ladder was located in the L-shaped goalbox. A correction procedure was employed throughout (after an error, the subject remained in the maze until finding the goal). The animals were given six trials per day with a 20 -min intertrial interval. On any given day, the right arm was correct for half of the subjects in each group and the left arm was correct for the remaining subjects.

\section{Results}

Only 1 infected mouse died following inoculation. Data for this animal were discarded. After 13 serial reversals, the subjects were performing at a stable rate such that the animals would generally make errors only on the first or second trial of the day. Figure 3 shows the percentage of errors on Trials 2-6 on the last training day and the test days postinfection. An ANOVA with treatment as a between-subjects effect and time period as a withinsubjects effect indicated that the effects of treatment $[F(1,13)=20.23, p<.001]$ and the interaction between treatment and time period $[F(6,78)=2.5, p<.05]$ were both significant. Post hoc analyses indicated that the groups did not differ before Day 7 and thereafter were significantly different.

Figure 4 shows the change in brain viral titers over time in a set of animals not trained on the discrimination task (4 animals per time period). Here it can be seen that virus titers peaked on Day 4 and thereafter declined. An
ANOVA on the $\log$ of the titer +1 indicated that the effect of time period was significant $[F(3,12)=9.30$, $p<.01]$. Although the data presented in Figures 3 and 4 were collected from different subjects, they indicate that the behavioral effects examined in this model began during the declining phase of virus growth.

\section{GENERAL DISCUSSION}

The effects of viral infection of the nervous system, or on any organ system for that matter, can be due to one of three processes. First are the direct effects of the presence of intracellular parasites on the host cell. The second process would be the local response to tissue destruction (e.g., the arachidonic acid cascade). Finally, a structural or functional change may occur as a result of the removal of the virus by the cellular or humoral immune response.

An example of a direct effect of viral infection would be the suggestion by Elizan, Maker, and Yahr (1983) that cell lysis is required to produce alterations in brain catecholamines following infection of mice with HSV. An

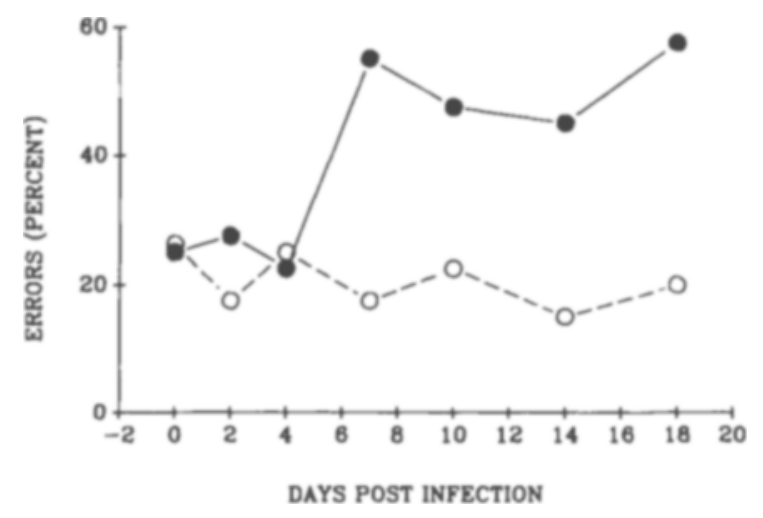

Figure 3. Performance on a serial spatial-reversal task in a water Y-maze by Balb/c mice infected with HSV (solid lines) and controls (dashed lines) as a function of days following inoculation.

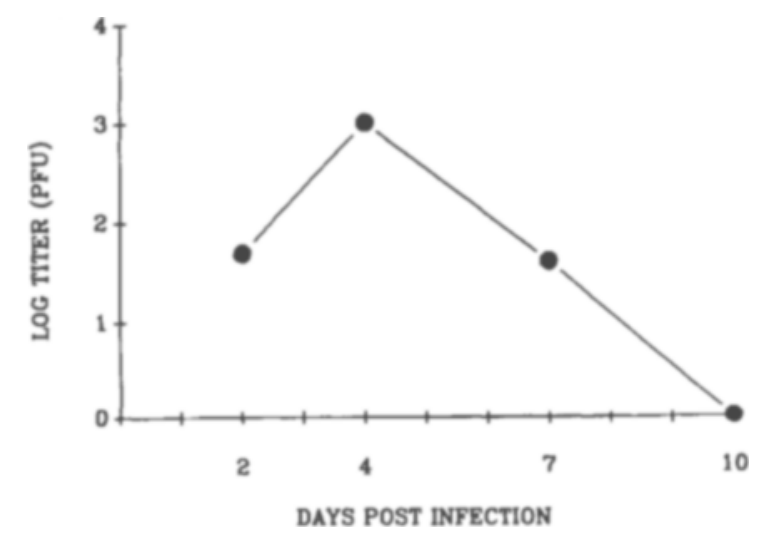

Figure 4. Brain virus titers in Balb/c mice infected with HSV as a function of days following inoculation. 
example of effects produced by a local response to infection would be the suggestion by Seegal and McFarland (1988) that a small focus of infection can produce an area of widespread inflammation, which would then produce an alteration in catecholamine levels. Both of these suggestions were applied to models using very virulent strains of HSV which produced death in a few days. An example of the third type of effect is suggested by Townsend (1985), who observed demyelination of the trigeminal root entry zone 7 days following corneal inoculation of HSV $1 \mathrm{in} \mathrm{Balb/c}$ mice. Since T cell-deficient mice showed less demyelination, it was suggested that the cellular immune response was involved in the pathological process. Thus, viral infection of the nervous system may produce functional changes by a direct effect of infection of cells, as a result of local processes such as an inflammatory response, or by the action of the cellular or humoral immune response.

Infection of mice with most laboratory strains of HSV results in brain virus titers that quickly reach levels several orders of magnitude greater than those observed in the present study. Consequently, a high degree of virulence is generally the rule (Dix et al., 1983). Such virulence in mice may possibly be due to alterations in the virus resulting from tissue culture passage (Costanzo et al., 1986; Rajcani \& Szanto, 1973). In any case, HSV is generally benign in the human population, and even given infection of the brain (Adams \& Victor, 1981), survival is higher than in most animal studies, where $100 \%$ mortality is often the rule. Since less virulent strains of HSV are available (Dix et al., 1983), the development of animal models characterized by higher survival rates may be most relevant to understanding pathogenesis in the human population. It is important to note that it is difficult to make precise quantitative comparisons between the human disease and animal models, however.

The results of the present study suggest that the behavioral effects of infection with HSV are associated with the period in time that coincides with a decline in brain virus titers. This was observed with two distinct host-virus strain combinations, both of which may be characterized by the fact that most of the animals survived infection. The behavioral effects associated with herpes simplex infection of mice have not previously been associated with the elimination of the virus from the brain. Lycke and Roos (1974) found increased levels of activity in mice as early as $\mathbf{2}$ days after infection, with death occurring as early as 4 days postinfection. Although brain viral titers were not reported in that study, the fact that the mice all died within a short period of time suggests that viral growth rates were very rapid, although factors other than growth rate could influence virulence. Thus, animal models of HSV encephalitis characterized by a rapid growth of the virus and a high degree of mortality may produce effects distinctly different from models of brain HSV infection in which virus growth rates are slow and survival is high.
Seegal and Hotchin (1978) observed reduced activity levels in response to amphetamine challenge in mice 3 days following intracerebral infection with HSV. To prevent high mortality rates, those mice also received a footpad immunization 2 weeks earlier. In that case, it is possible that the prior footpad immunization resulted in the more rapid involvement of cellular or humoral immunity in the pathological process. Alternatively, it may be the case that amphetamine challenge is more sensitive to the early features of HSV encephalitis.

Given the low virulence of the mouse-strain and virusstrain combinations examined in the present study, the results suggest that certain persistent behavioral effects may begin to appear as processes involved in the elimination of the virus from the brain are initiated. This follows from the association of these behavioral effects with the declining phase of the viral growth curve. This suggests that the immune response may have a role in the pathogenesis of herpes encephalitis. An alternative possibility is that the cumulative effects of cell lysis reach a level sufficient to produce behavioral effects coincidently at a time when brain virus titers are declining. Although this scenario is possible, it does seem unlikely that it would happen in both mouse-virus models.

The intracerebral inoculation technique used here does not represent the best model of human HSV encephalitis since microinjection of the virus produces characteristic focal lesions (McFarland, Sikora, \& Hotchin, 1986). However, this technique does allow for an examination of the dynamics of the development of the pathological process. Correlating behavioral change with virus growth is particularly well suited for examining this question because it involves an examination of a functional change with the net rate of virus growth.

\section{REFERENCES}

ADAms, R. D., Victor, M. (1981). Principles of neurology (2nd ed.). New York: McGraw-Hill.

Costanzo, F., Borgatti, M., Bartoletti, A. M., Fon-Tomasi, L., Cassai, E., Mannini-Palenzona, A. (1986). Further characterization of virus obtained from herpes simplex virus type 1 recurrences and primary infections: Influence of the temperature of incubation upon glycoprotein synthesis and vinus release. Archives of Virology, 88, 293-299.

Dix, R. D., McKendall, R. R., \& Baringer, J. R. (1983). Comparative neurovinulence of herpes simplex virus type 1 strains after peripheral or intracerebral inoculation in $\mathrm{Balb} / \mathrm{c}$ mice. Infection \& Immunity, 40, 103-112.

Elizan, T. S., Maker, H., \& Yahr, M. D. (1983). Neurotransmitter synthesizing enzymes in experimental viral encephalitis. Joumal of Neural Transmission, 40, 139-147.

Heeg, U., Dienes, H. P., Muller, S., FAlKe, D. (1986). Involvement of Actin-containing microfilaments in HSV-induced cytopathology and the influence of inhibitors of glycosylation. Archives of Virology, 91, 257-270.

Hierons, R., Janota, I., \& Corselus, J. A. N. (1978). The late effects of necrotizing encephalitis of the temporal lobes and limbic areas: A clinico-pathological study of 10 cases. Psychological Medicine, 8 , 21-42. 
LYCKE, E., \& Roos, B. E. (1974). Influence of changes in brain monamine metabolism on behavior of herpes simplex infected mice. Journal of the Neurological Sciences, 22, 277-289.

MCFarland, D. J., \& Hotchin, J. (1983). Host genetics and the behavioral sequelae to herpes encephalitis in mice. Physiology \& Behavior, 30, 881-884.

MCFaRLAND, D. J., Hotchin, J. (1987). Animal models in behavioral neurovirology. In E. Kurstak, Z. J. Lipowski, \& P. V. Morozov (Eds.), Viruses, immunity, and mental disorders (pp. 189-198). New York: Plenum.

MCFarland, D. J., Sikora, E., Hotchin, J. (1986). The production of focal herpes encephalitis in mice by stereotaxic inoculation of virus: Anatomical and behavioral effects. Journal of the Neurological Sciences, 72, 307-318.

Rajcani, J., \& Szanto, J. (1973). Pathogenicity of laboratorymaintained and low-passaged strains of herpes virus hominis in suckling mice: A comparative fluorescent antibody study. Acta Virologica, 17, 227-236.

Rubenstein, R., PrICE, R. W. (1983). Preservation of catecholamine uptake and release in herpes simplex virus type-1 infected PC12 cells. Journal of General Virology, 64, 2505-2509.

SeEgAL, R. F., \& Hotchin, J. (1978). Effects of herpes virus and amphetamine on locomotor activity. Birth Defects, 5, 179-184.

SEEGAL, R. F., McFarLand, D. J. (1988). Stereotaxic micro-injection of HSV-1 selectively decreases striatal dopamine concentrations in mice. Brain Research, 445, 234-240.

TownsEnd, J. J. (1985). Macrophage response to herpes simplex encephalitis in immune competent and $\mathrm{T}$ cell-deficient mice. Journal of Neuroimmunology, 7, 195-206.

(Manuscript received September 28, 1988; revision accepted for publication May $1,1989$.

\title{
Announcement
}

\author{
19th Annual Meeting of the Society for Computers in Psychology \\ Atlanta, Georgia \\ November 16, 1989
}

The 19th Annual Meeting of the Society for Computers in Psychology will be held at the Hyatt Regency Hotel in Atlanta, Georgia on November 16, 1989. As usual, this meeting will feature papers on applications of computers to all areas of psychology-experimental, clinical, and educational.

For further information, contact Paula Goolkasian, Department of Psychology, University of North Carolina at Charlotte, Charlotte, NC 28223. 